\title{
SHORT ARTICLES
}

\section{COMPARISON OF SEVERAL CULTURE MEDIA USED FOR STUDIES ON MYCOBACTERIOPHAGES}

\author{
C. E. Stager* and P. R. J. Gangadharam $\dagger \ddagger$ \\ * Mycobacteriology Laboratories, Jefferson Davis Hospital and \\ $\dagger$ Department of Pathology, Baylor College of Medicine, Houston, Texas 77025, USA
}

Plates XII-XIV

\begin{abstract}
MEDIA to be used for growing mycobacteria for mycobacteriophage studies must provide for exacting requirements. They must produce logarithmic growth of the host bacterium and provide a favourable environment for the attachment of the phage and eventual lysis of the host. Redmond and Ward (1966) developed a standard medium (RVA medium) for study of phage lysis of mycobacteria. Šula and Sulová (1969) developed a simple chemically defined medium (N-1 medium) for this purpose. They claimed that $\mathrm{N}-1$ medium was slightly better than RVA medium for phage lysis of mycobacteria. However they used only fastgrowing mycobacteria and paid particular attention to the lytic patterns produced by phage; in general their comparisons were not based on quantitative data. There have been no further comparisons of $\mathrm{N}-1$ medium with other media.

The ability of mycobacteria to reduce a tellurite salt solution to metallic tellurium was reported by Gosio (1905). The studies of Belfanti (1912) and Corper (1915) supported this finding, and Šula (1947) pointed out the usefulness of the method for early recognition of viable mycobacterial colonies. Sula and Sulová (1963) utilised tellurite reduction to study the patterns of phage lysis of mycobacteria.

The purpose of our study was to assess the value of five different media in facilitating $(a)$ mycobacteriophage infection, and $(b)$ lysis of both fast-growing saprophytic and slowgrowing pathogenic mycobacteria. We also attempted to assess the value of tellurite reduction in detecting plaques.
\end{abstract}

\section{MATERIALS AND METHODS}

Media. All media were adjusted to $p \mathrm{H} 7 \cdot 0( \pm 0 \cdot 2)$ and autoclaved at $121^{\circ} \mathrm{C}$ for $20 \mathrm{~min}$.

RVA Agar Base (Redmond and Ward, 1966; BBL), 7H10 Medium (Middlebrook and Cohn, 1958; Difco) and 7H11 Medium (Cohn, Waggoner and McClatchy, 1968; Difco) were reconstituted according to instructions, adding a supplement of glycerol $(0.5 \%)$ to the water. After autoclaving, the media were cooled to $47^{\circ} \mathrm{C}$ and admixed with oleic acid albumin dextrose complex $(10 \%)(\mathrm{OADC}$, Difco).

Sauton's medium and N-1 medium were prepared in the laboratory. Sauton's medium contained iron ammonium citrate $0.05 \mathrm{~g}$, dipotassium phosphate $0.5 \mathrm{~g}$, magnesium sulphate $0.5 \mathrm{~g}$, citric acid $2 \mathrm{~g}$, asparagine $4 \mathrm{~g}$, agar $20 \mathrm{~g}$, glycerol $40 \mathrm{ml}$ and distilled water to $1000 \mathrm{ml}$.

$\mathrm{N}-1$ medium (Sula, 1947) contained potassium mono-hydrogen phosphate $1.5 \mathrm{~g}$, magnesium sulphate $0.5 \mathrm{~g}$, asparagine $1.5 \mathrm{~g}$, glycerol $3.6 \mathrm{ml}$, agar $20 \mathrm{~g}$ and distilled water to $1000 \mathrm{ml}$.

Methods of study of phage lysis. Mycobacteriophages BGI, BKI, CRI-3, G37 and LG

Received 17 Sept. 1976; accepted 29 Oct. 1976.

$\ddagger$ Present address: National Jewish Hospital and Research Center, 3800 East Colfax Avenue, Denver, Colorado 80 206, USA.

Reprint requests to P. R. J. G.

J. MED. MICROBIOL.—VOL. 11 (1978) 
were propagated on Mycobacterium smegmatis strain 607B (referred to hereafter as 607B) and DS6A on Mycobacterium tuberculosis strain H37Rv (referred to hereafter as H37Rv).

Strain 607B was subcultured in Nutrient Broth (Difco) and incubated at $37^{\circ} \mathrm{C}$ for $48 \mathrm{~h}$ in a shaking waterbath. Strain H37Rv was subcultured in 7H9 Broth (Difco) containing Tween 80 (Difco) and OADC enrichment and was incubated at $37^{\circ} \mathrm{C}$ for 5 days in a shaking waterbath. Strains 607B and H37Rv were washed first with nutrient broth and then with 7H9 containing glycerol $(0.5 \%)$, before exposure to mycobacteriophage.

To test the lytic ability of phages on the various media, the latter were first flooded with the cultures of the appropriate strains; excess culture was removed with a Pasteur pipette, and the inoculated media were left at $37^{\circ} \mathrm{C}$ for $3 \mathrm{~h}$. They were then used as follows. (1) "Direct lysis" was determined by placing undiluted drops of phage stock preparation by means of Pasteur pipettes on the various media seeded with the appropriate strains. The plates were incubated at $37^{\circ} \mathrm{C}$ for $2-5$ days. (2) To find the routine test dilution (RTD) of a stock phage suspension, eight 10 -fold dilutions were made in nutrient broth. These were sampled with capillary pipettes, droplets being placed on each medium and allowed to dry at room temperature. The plates were incubated at $37^{\circ} \mathrm{C}$ and examined for plaque formation at 24-h intervals. The highest dilution to produce complete clearing was the RTD. (3) Counts of plaque-forming units (PFU) were obtained by the overlay method (Adams, 1959). Incubation was at $37^{\circ} \mathrm{C}$ for $2-5$ days. (4) The paper-disk method for studying phage lysis consisted of soaking blank antibiotic disks (Schleicher and Schuell, Inc., Keene, New Hampshire) with phage suspension at a dilution 100 times less than the RTD and placing the disk on lawns of the appropriate host mycobacterium (Gangadharam, Simmons and Stager, in preparation). The plates were incubated with the disks uppermost at room temperature for $3 \mathrm{~h}$ and then inverted before incubating at $37^{\circ} \mathrm{C}$ for $24 \mathrm{~h}$.

When potassium tellurite $\left(\mathrm{K}_{2} \mathrm{TeO}_{3}\right)$ was used, $3-5 \mathrm{ml}$ of a $1 \%(\mathrm{w} / \mathrm{v})$ solution were poured over the cultures on which either plaques or zones of lysis had developed. After $10 \mathrm{~min}$. the excess was removed and the plates were examined after $4 \mathrm{~h}$ and $24 \mathrm{~h}$ at room temperature $\left(25^{\circ} \mathrm{C}\right)$.

\section{RESULTS}

\section{Comparison of five culture media}

"Direct lysis". All media, except Sauton's, supported good direct lysis, regardless of the phage-host system employed. Fig. 1 shows the results with phages G37 and BKI on host 607B, as examples.

\section{TABLE I}

The routine test dilutions (RTD) of suspensions of six mycobacteriophages as measured on five different culture media

\begin{tabular}{|c|c|c|c|c|c|}
\hline \multirow{2}{*}{$\begin{array}{l}\text { Suspension } \\
\text { of phage }\end{array}$} & \multicolumn{5}{|c|}{$\begin{array}{c}\text { RTD values }\left(\log _{10}\right) \text { obtained on the following } \\
\text { media }\end{array}$} \\
\hline & RVA & N-1 & $7 \mathrm{H} 10$ & 7H11 & Sauton \\
\hline $\begin{array}{l}\text { G37 } \\
\text { BKI } \\
\text { BGI } \\
\text { LG } \\
\text { CRI-3 } \\
\text { DS6A }\end{array}$ & $\begin{array}{l}3 \\
1 \\
3 \\
2 \\
2 \\
4\end{array}$ & $\begin{array}{l}3 \\
4 \\
3 \\
4 \\
3 \\
4\end{array}$ & $\begin{array}{l}4 \\
4 \\
4 \\
4 \\
4 \\
4\end{array}$ & $\begin{array}{l}3 \\
4 \\
4 \\
4 \\
3 \\
4\end{array}$ & $\begin{array}{l}1 \\
\frac{1}{二} \\
=\end{array}$ \\
\hline
\end{tabular}

$\overline{-}=$ No lysis detected.

Phage DS6A was specific for $M$. tuberculosis strain H37Rv; the other five phages were specific for M. smegmatis strain 607B. 
MEDIA FOR STUDIES ON MYCOBACTERIOPHAGES

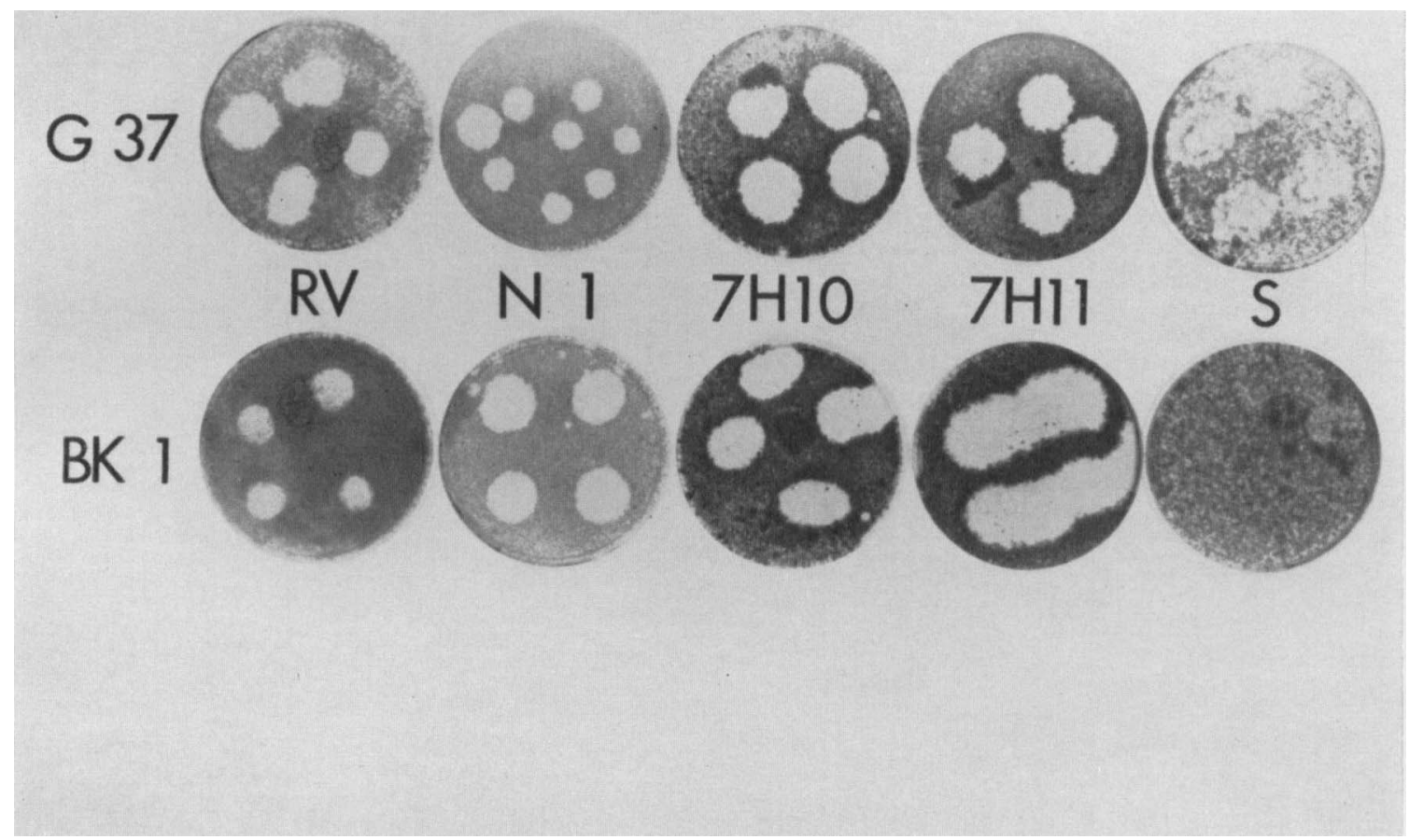

FIG. 1.-Comparison of five media in respect of " direct lysis " produced by phages G37 and BKI on Mycobacterium smegmatis strain 607B. Satisfactory lysis was produced on RVA, N-1, 7H10 and 7H11 media, but not on Sauton's medium (S). 
MEDIA FOR STUDIES ON MYCOBACTERIOPHAGES

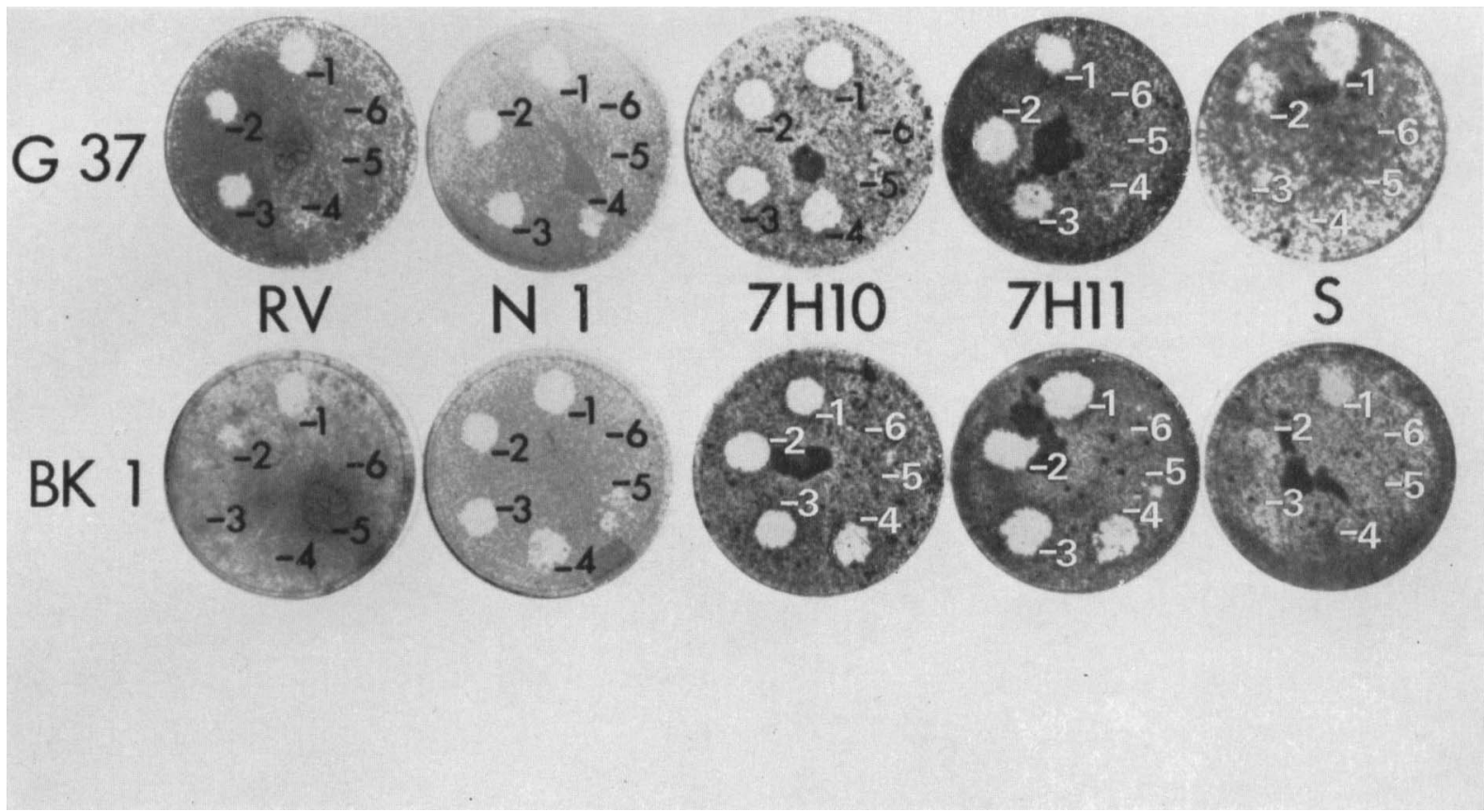

Fig. 2.-Comparison of five media in respect of their suitability for determining the routine test dilution (RTD). Mycobacterium smegmatis strain 607B and its phages G37 and BKI were used. Numerals represent the dilutions $\left(\log _{10}\right)$ of phage suspension. Note that higher RTD values were obtained on $\mathrm{N}-1,7 \mathrm{H} 10$ and $7 \mathrm{H} 11$ media than on RVA medium, especially when phage BKI was used. Sauton's medium (S) was inferior. 


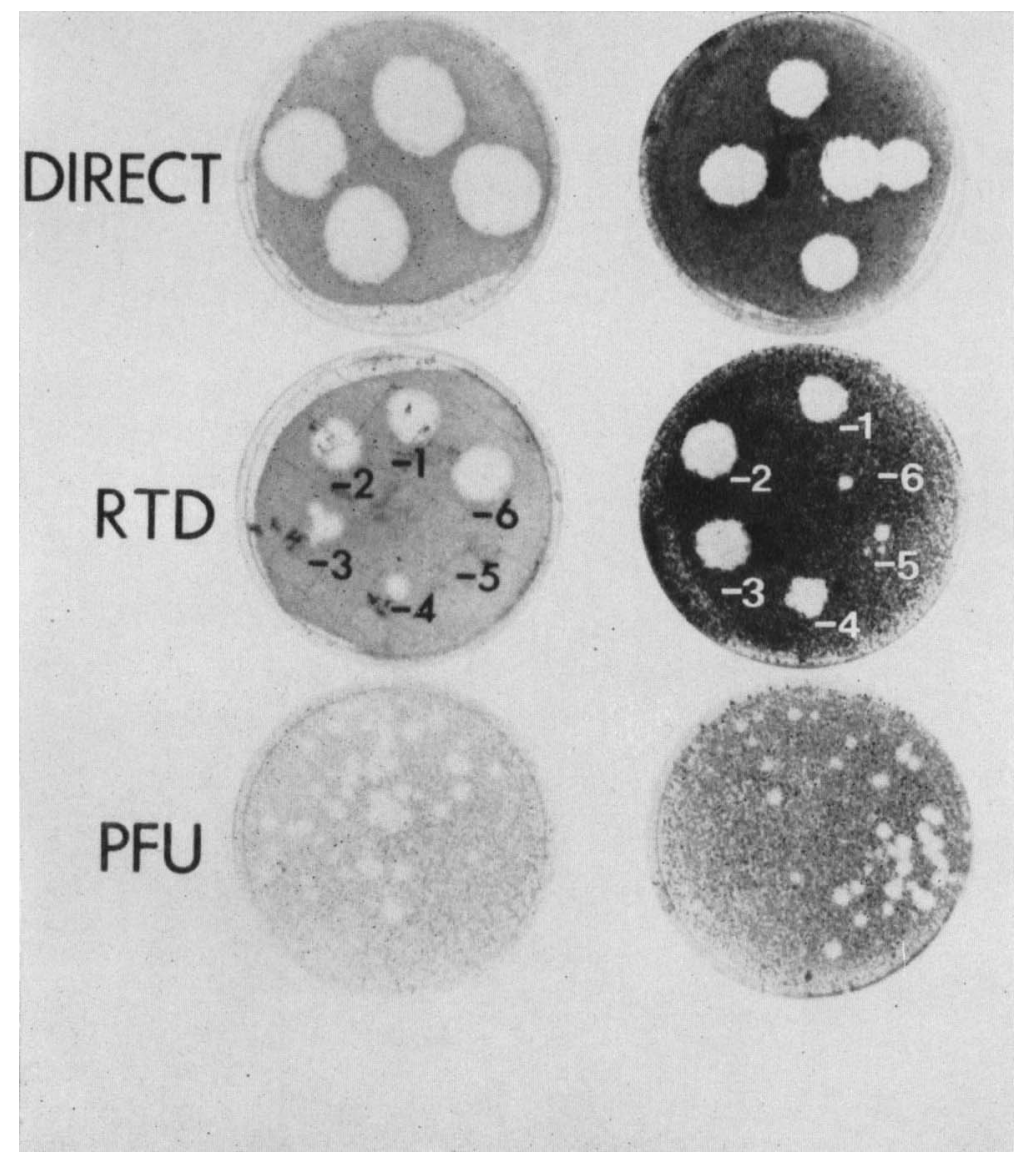

FIG. 3.-Comparison of untreated (left) and tellurite-treated (right) plates in respect of the lysis produced by the three methods: (1) "direct lysis", (2) routine test dilution (RTD), and (3) plaque-forming unit (PFU) count. Mycobacterium smegmatis strain 607B and phage LG were used on N-1 medium. Numerals represent the dilutions $\left(\log _{10}\right)$ of phage suspension. 
TABLE II

The counts of plaque-forming units (PFU) in suspensions of six mycobacteriophages as measured on five different culture media

\begin{tabular}{|c|c|c|c|c|c|}
\hline \multirow{2}{*}{$\begin{array}{l}\text { Suspension } \\
\text { of phage }\end{array}$} & \multicolumn{5}{|c|}{$\begin{array}{c}\text { PFU per ml ( } \log _{10} \pm \text { standard deviation) obtained on the following } \\
\text { media }\end{array}$} \\
\hline & RVA & $\mathrm{N}-1$ & $7 \mathrm{H} 10$ & $7 \mathrm{H} 11$ & Sauton \\
\hline $\begin{array}{l}\text { G37 } \\
\text { BKI } \\
\text { BGI } \\
\text { LG } \\
\text { CRI-3 } \\
\text { DS6A }\end{array}$ & $\begin{array}{l}9 \cdot 32 \pm 0.22 \\
9 \cdot 34 \pm 0.87 \\
9 \cdot 69 \pm 0 \cdot 10 \\
7 \cdot 07 \pm 0.50 \\
9 \cdot 11 \pm 0.35 \\
7 \cdot 14 \pm 0.48\end{array}$ & $\begin{array}{c}9 \cdot 65 \pm 0.36 \\
0.59 \pm 0.30 \\
9 \cdot 37 \pm 0.14 \\
7.69 \pm 0.20 \\
8.84 \pm 0.50 \\
=\end{array}$ & $\begin{array}{l}9 \cdot 51 \pm 0.42 \\
9 \cdot 11 \pm 0.47 \\
9 \cdot 97 \pm 1 \cdot 69 \\
7 \cdot 49 \pm 0.46 \\
9 \cdot 11 \pm 0.22 \\
7.68 \pm 0.22\end{array}$ & $\begin{array}{l}9 \cdot 55 \pm 0.42 \\
9 \cdot 17 \pm 1 \cdot 04 \\
9 \cdot 80 \pm 0 \cdot 10 \\
7 \cdot 55 \pm 0 \cdot 10 \\
9 \cdot 20 \pm 0 \cdot 10 \\
8 \cdot 11 \pm 0 \cdot 17\end{array}$ & $\begin{array}{l}= \\
= \\
=\end{array}$ \\
\hline
\end{tabular}

$-=$ No plaques detected.

Phage DS6A was specific for $M$. tuberculosis strain H37Rv; the other five phages were specific for $M$. smegmatis strain 607B.

Routine test dilution. Table I shows a comparison of the RTD of the six phages on their respective hosts, as tested on the various media. The experiment was run in duplicate on two separate occasions with consistent results. Phages specific for 607B usually had a higher RTD on $\mathrm{N}-1,7 \mathrm{H} 10$ and 7H11 media than on RVA medium, 7H10 being generally the best. However, DS6A, specific for H37Rv, had an identical RTD on RVA, N-1, 7H10 and 7H11 media; plaques were more easily seen on RVA medium than on the other media. Sauton's medium was inadequate, regardless of the phage-host system employed. Fig. 2 shows the RTD of phages G37 and BKI on host 607B, as examples. It will be noted, particularly with phage BKI, that the RTD was higher on $\mathrm{N}-1,7 \mathrm{H} 10$ and $7 \mathrm{H} 11$ media than on RVA medium.

Counts of plaque-forming units. Table II shows an experiment to compare the PFU counts of the various phages on their hosts, as tested on the five media. The experiment was made in duplicate, and further duplicate experiments were carried out on a later occasion; all four experiments gave similar results. The PFU counts for each phage specific for 607B were not significantly different on RVA, N-1, 7H10 and 7H11 media. The PFU counts for DS6A were not significantly different on RVA, 7H10 and 7H11 media, but $\mathrm{N}-1$ medium did not support growth of the host strain H37Rv.

\section{Potassium tellurite as an aid in detecting phage lysis}

Phage lysis of host strain $607 \mathrm{~B}$. The direct lysis, RTD and PFU methods all resulted in readily discernible lysis of $607 \mathrm{~B}$ by its five phages, but the use of tellurite made the lysis produced by PFU even easier to detect. Representative results obtained with 607B and phage LG on N-1 medium are shown in fig. 3.

Phage lysis of host strain H37Rv. The direct lysis and RTD methods resulted in readily discernible lysis of H37Rv by phage DS6A on RVA, N-1, 7H10 and 7H11 media, but the use of tellurite gave improved contrast. The faintly discernible lysis produced by PFU on RVA, $7 \mathrm{H} 10$ and $7 \mathrm{H} 11$ media was greatly enhanced by the use of tellurite. It was found that $\mathrm{N}-1$ medium did not suppprt the growth of H37Rv in the overlay technique used for PFU counts.

Lytic zones produced by phage-impregnated paper disks. Tellurite reduction was studied, on RVA medium only, with host strain 607B and phages BGI, BKI, CRI-3, G37 and LG. The lytic zones were readily discernible both with and without the addition of tellurite.

With all phage-host systems studied, the effects of tellurite reduction were optimal at $24 \mathrm{~h}$.

\section{Discussion}

Suspensions of mycobacteriophages specific for host 607B usually had a higher RTD on $\mathrm{N}-1,7 \mathrm{H} 10$ and 7H11 media than on RVA medium (table 1), but the PFU counts obtained on 
RVA medium were not significantly different from those obtained on the other three media (table II). The results obtained with the host strain H37Rv and its phage DS6A showed that the RTD on RVA medium was identical to that on N-1, 7H10 and 7H11 media. The plaques were more easily discernible on RVA medium than on the other media because of the heavier growth of the lawn of mycobacteria. The PFU counts obtained on RVA, 7H10 and 7H11 media were not significantly different, but no plaques developed on $\mathrm{N}-1$ medium when the overlay technique was used. The value of RVA medium as compared with that of other media depended both on the mycobacterial strain and on the technique used. The reasons for this are not clear, but it seems probable that RVA-a very rich medium-stimulated the growth of 607B to a far greater degree than did the other media, especially under the aerobic conditions used in determining the RTD. Such growth might produce numbers of bacteria far in excess of the available phage particles. The overlay technique used in determining the counts of PFU may have resulted in conditions that were less than completely aerobic and these may have suppressed partially the growth of 607B. The growth-stimulating properties of RVA medium may have played a less significant part in respect of $H 37 R v$, an organism that grows more slowly than 607B. Under the aerobic and micro-aerophilic conditions created by the RTD and PFU tests respectively, all three media (RVA, 7H10 and 7H11) may be equally effective in promoting the growth and susceptibility to lysis of the host organism.

Tellurite reduction, after $24 \mathrm{~h}$ at room temperature, was useful in making lysis more easily discernible; this statement is based on the use of the rapidly-growing saprophyte, 607B, and the slowly-growing pathogen, H37Rv, and on tests made by the direct lysis, RTD, PFU and disk-methods. However, the main value of tellurite reduction was in detecting plaques arising from PFU in the overlay technique. Such plaques were otherwise often barely discernible.

\section{SUMMARY}

The value of RVA, N-1, 7H10, 7H11 and Sauton's media for studies on mycobacteriophage infection and lysis of mycobacteria was assessed. Experiments were made with mycobacteriophages BGI, BKI, CRI-3, G37, and LG, all of which lyse Mycobacterium smegmatis strain 607B, and with mycobacteriophage DS6A which lyses Mycobacterium tuberculosis strain H37Rv. The methods involved "direct lysis", the measurement of " routine test dilutions" and counts of plaque-forming units. It was found that $\mathrm{N}-1,7 \mathrm{H} 10$ and $7 \mathrm{H} 11$ media gave better overall results than RVA medium for $M$. smegmatis strain 607B and its phages, and that RVA medium was generally the most useful for $M$. tuberculosis strain H37Rv and its phage. Sauton's medium was inadequate for all the phage-host systems employed.

This investigation was supported in part by the Kelsey-Leary Foundation, Houston, Texas.

\section{REFERENCES}

Adams, M. H. 1959. Bacteriophages, New York.

BelfantI, S. 1912. Die vitale reaktion nach Gosio beim tuberkelbazillus. Z. Chemother., 1, Orig., 113.

CoHn, M. L., Waggoner, R. S. AND MCClatchy, J. K. 1968. 7H11 Medium for cultivation of mycobacteria. Am. Rev. resp. Dis., 98, 295.

CORPER, H. H. 1915. Sodium tellurite as a rapid test for the viability of tubercle bacilli. Studies on the biochemistry and chemotherapy of tuberculosis. J. infect. Dis., 16, 47.

Gosio, B. 1905. Indicatoren des bakterienlebens und ihre praktische bedeutung. Z. Hyg. InfektKrankh., 51, 65.

MiddlebrooK, G. AND CoHN, M. L. 1958. Bacteriology of tuberculosis. Laboratory methods. Am. J. publ. Hlth, $48,844$.

REDMOND, W. B. AND WARD, D. M. 1966. Media and methods for phage-typing mycobacteria. Bull. Wld Hlth Org., 35, 563. 
Šlul, L. 1947. The reduction of potassium tellurite by Mycobacterium tuberculosis. Am. Rev. Tuberc., 56, 241.

Šula, L. AND ŠUlová, J. 1963. The tellurite zonal phenomenon of mycobacterial lysis observed in a BCG strain exposed to mycophage My $\mathrm{F}_{2} \mathrm{P} / 59$. Am. Rev. resp. Dis., 88, 77.

Šula, L. AND Šulová, J. 1969. A comparative study of special agar medium (Redmond) and simple agar medium for the phage-typing of mycobacteria. Bull. Wld Hlth Org., 41, 17. 